\title{
Organoid research in digestive system tumors (Review)
}

\author{
XIAOXIAO YANG ${ }^{*}$, XUEWEN XU* ${ }^{*}$ HAITAO ZHU, MING WANG and DONGQING WANG \\ Department of Radiology, The Affiliated Hospital of Jiangsu University, Zhenjiang, Jiangsu 212031, P.R. China
}

Received August 14, 2020; Accepted February 4, 2021

DOI: 10.3892/ol.2021.12569

\begin{abstract}
Digestive system tumors are the most common cause of cancer-associated mortality worldwide, although their underlying biological behavior still requires further investigation. Most of the in vitro studies that have been published have been based on the two-dimensional (2D) culture system. However, digestive system tumors exhibit considerable histological and functional heterogeneity, and clonal diversity and heterogeneity cannot be entirely reflected in the $2 \mathrm{D}$ culture system. Recently, the development of organoids appears to have shed some light on this area of cancer research. The present review discusses the recent advancements that have been made in the development of several specific organoids in digestive system solid tumors.
\end{abstract}

\section{Contents}

1. Introduction

2. Development and merit of organoids

3. Application of organoids in digestive system solid tumor cancer research

4. Application of organoids in digestive system solid tumors

5. Summary and outlook

\section{Introduction}

The human digestive system is composed of a digestive tube (comprising the oral cavity, pharynx, esophagus, stomach, small intestine and colorectum) and digestive gland organs, including salivary glands, liver, gallbladder and pancreas (1). The most common malignant tumors of the digestive system

Correspondence to: Miss Ming Wang or Professor Dongqing Wang, Department of Radiology, The Affiliated Hospital of Jiangsu University, 438 Jiefang Road, Zhenjiang, Jiangsu 212031, P.R. China E-mail: wangm0330@163.com

E-mail: wangdongqing71@163.com

*Contributed equally

Key words: organoid, three-dimensional culture system, cancer research, drug screening, personalized treatment include esophageal cancer, gastric cancer, colorectal cancer, liver cancer and pancreatic cancer (1). Due to the high incidence rate of digestive system cancers, digestive tract tumors accounted for $43.3 \%$ of cancer incidence in China from 2000-2015, a comprehensive study of their underlying molecular biology would cast light on effective methods of treatment (2). However, an accurate in vitro research model that would improve our understanding of the complete picture of tumor development is currently lacking.

Current in vitro cancer research models mainly focus on two-dimensional (2D) models, namely, tissue-lice cultures and 2D cell-line cultures (1). Tissue section models can capture transient interactions between physiologically relevant cellular tissues, although they often lose their phenotype quickly and are difficult to maintain for longer time periods (3). Currently, the most widely used tumor research model is based on patient-derived cancer cell lines (PDCs); however, although PDC culture models are economical, simple and the cell lines proliferate infinitely, they still have serious defects, such as the loss of genetic phenotype and heterogeneity, the lack of immune microenvironment and vascular network system, and cannot reproduce the morphology and function of the original tumor tissue (4).

Organoids are derived from multipotent tissue progenitors (adult stem cells) and cancer cells, consisting of multiple cell types with remarkable self-renewal and self-organizing abilities, which maintain the key structural and functional properties of organs $(5,6)$. The organoid model is more representative of typical physiological conditions compared with other models (4). Currently, the organoids for normal tissues, including the stomach (7), small intestine (8), liver (9), pancreas (10) and prostate (11), have been established successfully. Compared with PDCs, the patient-derived organoids (PDOs) not only reflect histopathological characteristics and the genomic and transcriptomic profiles of the original tumor, but also recapitulate the components of the tumor microenvironment (12). With the development of gene-editing technologies, including gene knockdown, gene overexpression and mutations, the successful establishment of organoids is of great significance for the study of solid tumors. In addition, organoids only require a small piece of tissue containing stem cells obtained from biopsy in the patient, and the injury inflicted on the patient is minimal (13).

The present review summarizes the currently developed digestive system solid tumor organoids and discusses their potential applications in future research. 


\section{Development and merit of organoids}

In the 1960s, three-dimensional (3D) culture models were developed, although these were mainly used to simulate the processes and investigate the molecular mechanisms of tissue and organ formation in vitro (14). In 2009, Sato et al (15) were the first to produce an organoid with small intestinal villi and crypt structure from adult stem cells. Since then, tissue culture has regained its status as a leading contemporary technique, as it was ranked as one of the top 10 breakthroughs in 2013 and 2017 by Science and Nature Methods magazines, respectively. As the technology has advanced, organoids have been extensively used as a powerful tool of tumor research, particularly regarding the digestive system (Fig. 1) (7-11).

Tumor organoid construction is associated with significantly higher success rates and lower costs compared with traditional 2D culture and tumor tissue xenotransplantation, which is convenient for gene modification and large-scale drug screening (1). In addition, the organoid model retains the tissue characteristics of the tumor during the experimental process, which is useful in terms of tumor microenvironment research, and provides a more realistic environment for tumor drug development (Table I) (2-6). Neal et al (16) developed PDOs using air-liquid interface technology. The PDOs model successfully retained the same intrinsic fibrous matrix and diversity of immune cell components that were observed in the original tumor tissues, which was confirmed at the genetic level. Furthermore, tumor-infiltrating lymphocytes in PDOs accurately retained the $\mathrm{T}$-cell receptor profile in the original tumor.

\section{Application of organoids in digestive system solid tumor cancer research}

Digestive system solid tumors accounted for $\sim 35.4 \%$ of cancer mortality rates worldwide in 2019 (17). The growth condition flexibility of organoids enable them to precisely simulate heterogeneity and pathophysiology of digestive system solid tumors, which is useful for gaining a better understanding of the biological behaviors of the disease (4). In addition, the introduction of gene-editing technology for organoids has exhibited great advantages in terms of studying the pathogenesis of digestive system solid tumor treatment (12). Phenotypical and genotypical analyses of PDOs have confirmed that these are highly similar to the original tumor (12). The molecular profile of tumor organoids was demonstrated to match the results of drug screening, indicating that PDOs can complement existing methods to identify cancer weaknesses and improve the treatment response. Thus, it is reasonable to speculate that, in the future, organoids will serve important roles in drug development, personalized therapy, gene therapy and regenerative medicine (Table II).

Mechanism of tumor development. From the onset of the mutant single cell to the progression of the lesion, the way the tumor develops and deteriorates is an interrelated and multistep process (5). It is important to study the exact mechanism underpinning each step in the overall process of tumor development and progression. Organoids consist of multiple cells that specifically mimic the microenvironment of cancer development (18-20). How to construct models in vitro at different stages of tumorigenesis and development is one of the key research areas of organoids (18). Bartfeld et al (19) developed p53 gene-mutation gastric cancer organoids with Nulin-3-selective medium, which revealed that Nutli3 can markedly inhibit the proliferation of normal gastric organoids, and has no effect on gastric organoids, which indirectly suggests that the occurrence and development of gastric cancer may be studied in vitro. Other researchers have also used gene-editing technology to mutate certain genes in normal gastric organoids to simulate the evolutionary process of gastric cancer, suggesting that deletion of p53 or mutation of KRAS contribute to the occurrence of gastric cancer (20).

Drug screening and personalized treatment. Organoids can proliferate infinitely in vitro, which maximizes the characteristics of cancer cells without inducing novel mutations during long-term culture (21). Thus, they provide an ideal model for drug testing and screening, which has good prospects in terms of the applicability of the technique in combination with traditional treatment methods, and this should prove useful in treating tumors (21). Based on the organoids and high-content screening technologies, Wenzel et al (22) screened out nine substances from two commercially available drug libraries that specifically target dormant cancer cells that are predominantly located in the internal core area of the tumor tissues, and are resistant to treatment with conventional chemotherapy or radiotherapy. Arena et al (23) suggested that one colorectal cancer subpopulation with poor prognosis and limited therapeutic options may be sensitive to poly(ADP-ribose) polymerase inhibitors, and drug-screening assays based on PDOs may be used to screen populations of cells that are sensitive to olaparib. In addition to drug screening, Yao et al (24) constructed a PDO biobank from patients with locally advanced rectal cancer (LARC). The PDOs were able to identify patients with LARC that were sensitive to neoadjuvant chemoradiation, thereby improving the treatment of this disease, highlighting that PDOs may be used to predict chemotherapy or radiotherapy responses. From the perspective of personal precision treatment, organoids can be used for expanding limited cancer samples from patients, for the screening of drugs, and as a therapeutic avenue for specific patients, assessing the side-effects of drugs and therapies in patients (25). In summary, organoids provide helpful assistance in terms of the accurate medical treatment of individual patients with digestive system solid tumors.

The malignant tumor organoid biological bank. The Malignant Tumor Organoid Biological bank is a biological sample bank for malignant tumors that combines targeted genes with drug action, thereby providing a basis for risk stratification and individualized treatment options (26). In 2015, the Sanger and the Hubrecht Institutes established the first living organoid biobank (27). The researchers involved examined 83 different types of experimental drugs and cancer drugs, and concluded that organoids with different genetic backgrounds are associated with different drug sensitivities.

\section{Application of organoids in digestive system solid tumors}

Liver cancer-derived organoid. According to the results of a 2019 survey, liver cancer is the second most lethal cancer 


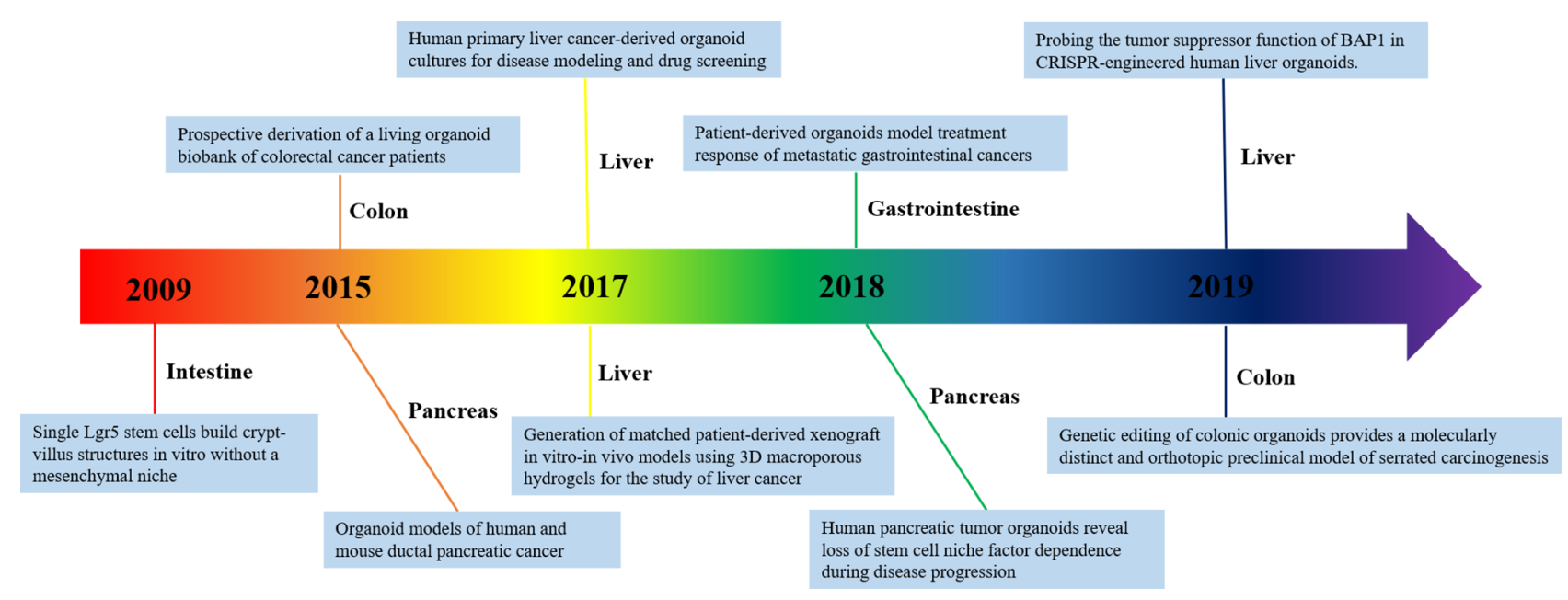

Figure 1. Timeline of organoids research in digestive system.

worldwide among men (17). 2D culture systems fail to preserve the histological architecture and gene expression patterns of the original tumor, and are unable to mimic the real communication between cancer and stomal cells (28). To better understand the processes of tumorigenesis and pathogenesis, and to develop curative treatments, organoids provide the most precise model for accurately simulating tumor biology behavior in patients (28).

Researchers at Cambridge University were the first to develop a mini tumor called a 'tumoroid' $(<0.5 \mathrm{~cm})$ to imitate three of the most common subtypes of primary liver cancer, namely hepatocellular carcinoma (HCC), cholangiocarcinoma (CC) and combined $\mathrm{HCC} / \mathrm{CC}$ (CHC) tumors (29). The tumoroid panel was used to screen 29 specific liver cancer target drugs (29). One of the screened drugs was able to specifically inhibit the activity of extracellular signal-regulated kinase (29). The ERK inhibitor SCH772984 markedly inhibited the growth of liver tumors in mice and beneficial therapeutic effects on three types of liver cancers were observed, suggesting its potential therapeutic value for primary liver cancer (29). This discovery is now considered to be the milestone for the usage of organoids in liver cancer research (29). Scientists from the National University of Singapore later designed a novel method to culture cancerous hepatocytes obtained from HCC-patient-derived xenografts (HCC-PDXs) for drug screening (30). To maintain the exact shape and function of hepatocytes so that they grew like tumor organoids, cells were cultured on a 3D macroporous cellulosic sponge system with optimized biochemical and mechanical properties (30). With the development of this technology, engineered organoids have continued to attract great attention, and the most attractive advantage is high-throughput drug screening (30). The genetic characteristics and heterogeneity of these models may make it possible to radically alter the screening and development of HCC drugs (9). Using CRISPR/Cas9 to study the role of mutated genes in the formation and progression of liver tumors, researchers have demonstrated that BAP1-mutated organoids have characteristics that distinguish them from healthy organoids (9). BAP1-mutated organoids have been reported to grow faster, move more easily and fuse more easily with other organoids, and these features are similar to aggressive malignancies (9). The morphological and behavioral changes of the organoid were reversed following introduction of unmutated BAP1 (9). Due to the ease with which this organoid permitted the function of mutated genes to be analyzed, its suitability for use in a wider context in the study of liver cancer was demonstrated (9).

Due to a lack of effective treatments for liver cancer, the development of liver cancer-derived organoids may accurately reflect the characteristics of this disease and the surrounding environment, which may facilitate the selection of more clinically efficacious drugs, thereby saving on costs and improving clinical transformation efficiency.

Pancreatic cancer-derived organoids. Pancreatic cancer is known as 'the stubborn bastion of 21st century medicine', with a 5 -year survival rate $<8 \%$ (31). To better understand pancreatic cancer development and progression, it is necessary to isolate cancer cells for analysis from the primary tumor tissue. However, nearly $85 \%$ of patients with pancreatic cancer will have already progressed to an advanced stage at the time of diagnosis, and thus are not suitable for surgery (32). Both normal cells and cancerous cells cultured in the laboratory were derived from a suboptimal environment that failed to reflect the important characteristics of tumor cells (12). The limited treatment options available for patients with pancreatic cancer has adversely affected their prognosis. Thus, 3D culture strategies of organoids can shed light on this disease.

In 2015, the Cold Spring Harbor Laboratory (CSHL) successfully established pancreatic organoids from murine and human tissues (33). The organoid was able to cultivate murine and human pancreatic cancer tissues (33). In addition, following transplantation into mice, the organoid was demonstrated to accurately imitate the pathogenesis of pancreatic ductal adenocarcinoma (PDA) and successfully recapitulate the full spectrum of tumor development (33). The identified molecular pathways based on the organoid specifically reflected the progression of the disease and provided the therapeutic target (34). Huang et al (35) established exocrine progenitor organoids derived from human pluripotent stem cells. These researchers developed five tumor organoids and tested their response to gemcitabine and epigenetic inhibitors. The results 
Table I. Characteristics of tissue sections, 2D culture cell lines and 3D organoids.

\begin{tabular}{lcccc}
\hline Variable & Initiation efficiency & Heterogeneity & Genetic manipulation & Drug screens \\
\hline Tissue & Low & High & Not amenable & Low-throughput \\
2D culture cell & Low & Low & Amenable & High-throughput \\
3D organoids & High & High & Amenable & High-throughput \\
\hline
\end{tabular}

2D, two-dimensional; 3D, three-dimensional.

Table II. Characteristics of tissue sections, 2D culture cell lines and 3D organoids.

\begin{tabular}{lll}
\hline Application & \multicolumn{1}{c}{ Cancer } & Refs. \\
\hline Tumor development mechanism & Gastric cancer, pancreatic cancer, colorectal cancer & $(9,19,20,33,36,39,46)$ \\
Drug screening and personalized treatment & Liver cancer, colorectal cancer & $(23,24,29,30)$ \\
Malignant Tumor Organoid Biological Bank & Colorectal cancer, gastric cancer & $(27)$ \\
\hline
\end{tabular}

2D, two-dimensional; 3D, three-dimensional.

demonstrated that the response of different organoids to the drugs varied and was positively correlated with drug-resistant biomarkers, findings that were consistent with the clinical response of patients. Using a small sample, pancreatic cancer organoids can retain the sensitivity of the patient's tissue to novel agents in vitro, which indicates the feasibility of using organoid-based techniques to study tumor heterogeneity (35). The greatest advantage of this technology is being able to establish a living sample library using multiple patient tumor tissues, and to study the specific pathogenesis of each patient, both of which are beneficial for targeted research and for rapid individualized drug testing (35).

Using tumor and normal tissue organoids from patients with pancreatic cancer, Chio et al (36) reported that the expression level of nuclear factor erythroid 2-related factor 2 (NRF2) was higher in the tumor organoids. NRF2 knockdown exhibited a different response since tumor organoids were not able to grow; however, the normal tissue organoids were not affected. Furthermore, this group studied changes in the oxidation level in the cells and confirmed that NRF2 exerts a notable effect on the proliferation of pancreatic tumor cells, suggesting that decreasing the levels of antioxidants kills tumors.

Researchers from the CSHL established the organoid based on cancer-associated fibroblasts (CAFs) and cancer cells from the patient tumor samples (37). The results demonstrated that myofibroblastic CAFs (myCAFs) higher levels of $\alpha$-smooth muscle actin ( $\alpha$ SMA), which approximated to the behavior of tumor cells in human and mouse tumor tissues. The researchers also discovered that co-culture resulted in the formation of dense matrix tissue. Inflammatory CAFs lacked elevated $\alpha$ SMA expression, which synthesizes and secretes interleukin 6 (IL-6), and is relatively distant from cancer cells in human and mouse PDA tumors (37). A previous study demonstrated that IL-6 is associated with the processes of cancer cell proliferation and cachexia in numerous patients with pancreatic cancer (38). This study reported that the behavior of stromal tissue in PDA is not uniform, which provides an opportunity to develop therapeutic drugs that can specifically target the stromal cells. Using the pancreatic organoid technique, this research group confirmed that FOXA1 activation induces the reprogramming of enhancers, which causes pancreatic cancer cells to metastasize (39).

CSHL also demonstrated that it is feasible to cultivate organoids using endoscopic ultrasonography (EUS)-derived pancreatic tumor samples (40). PDA organoids derived from the EUS samples can be developed within 2 weeks, with a success rate of $87 \%$. Establishment of PDA organoid lines for $\geq 5$ passages or cycles of growth (P5) was also demonstrated, with a success rate of $66 \%$ (40). The advent of EUS-guided fine-needle biopsy (EUS-FNB) technology may be used in the chest, abdomen and pelvis, and in the future, this technique is likely to be used to prepare organoids from a variety of different tumors for research purposes (40).

In addition to demonstrating the success of organoid development and the consistency between the organoids and the original tumors, in terms of pathology and genetics, Seino et al (41) also used organoids for tumor microenvironment research. They identified an association between the degree of malignancy of pancreatic adenocarcinoma (PDAC) and growth factors in the microenvironment, and demonstrated the association between GATA- 6 and Wnt in the niche. As CAFs provide a Wnt niche for PDAC, the supportive role of CAFs in pancreatic cancer was further confirmed (41). Their experimental results were verified using CRISPR-Cas9 technology. The concept of 'engineering organoids' in the article was innovative (41). Their research enabled pancreatic cancer to be classified as Wnt- and R-Spondin-dependent, Wnt-dependent, and Wnt and R-Spondin-independent (41). This classification is of great value in clinical practice and may be used for individual treatment (41).

Organoid technology not only has the potential to fill in the void of cancer genetics, but it also provides theoretical guidance for pancreatic cancer (42). The characteristics of pancreatic cancer organoids include maintaining the differentiation state 
tissue structure and phenotypic heterogeneity of the primary pancreatic cancer (42). Thus, organoid technology allows a new research direction for studying the biological characteristics of pancreatic cancer, enabling more effective anti-pancreatic cancer drugs to be investigated and the formulation of personalized treatment plans.

Gastrointestinal cancer-derived organoid. The first human colonic organoid model was achieved at Cincinnati Children's Hospital Medical Center using pluripotent stem cells (43). This technique has enabled the use of a human-like model to perform unprecedented research on colonic diseases, which will prove to be invaluable in the future. Transforming growth factor- $\beta$ (TGF- $\beta$ ) can promote the proliferation and distant metastasis of colorectal cancer cells, and induce fibroblasts to differentiate into myofibroblasts, which promotes the metastasis of colorectal cancer (44). Using the colorectal cancer organoids, Usui et al (45) concluded that fibroblasts secrete TGF- $\beta$. Utilizing combined organoids and CRISPR/Cas9 genetic engineering technology, Australian scientists demonstrated that elevated expression levels of v-raf murine sarcoma viral oncogene homolog B1 were involved in the progression of serrated colorectal cancer (46).

Organoid models can also be applied in the case of genetic manipulation in gastrointestinal cancer (47-49). Nadauld et al (47) demonstrated that TGF- $\beta$ receptor 2 was able to inhibit the metastasis of gastric cancer based on the organoids in vitro, which was consistent with the results demonstrated in vivo with cadherin $1^{-/ /} / \mathrm{Tp} 53^{-/-}$gastric cancer mice (47). In examining mutations of the genes, Apc, Tp53, Kras and Smad4 in gastrointestinal organoids, Li et al (48) reported that they exhibited aggressive histological features on subsequent living transplants. Another research group constructed the largest known number of gastric cancer organoid biobanks, and considered in depth the genomic variation, molecular typing, chemosensitivity and sensitivity of targeted therapies for gastric cancer (49). This study is of great value in terms of comprehending the pathogenesis of gastric cancer, and in promoting the development of novel drugs for gastrointestinal cancer-targeted therapy.

The metastatic organoid model has rarely been constructed. The application of PDO-tested cancer drugs may assist specialists in treating patients with more personalized therapies specifically targeted for tumor metastasis (50). Researchers successfully cultivated into organoids 71 colorectal cancer, gastric cancer and other digestive system cancers with metastatic tumor samples from patients, and subsequently screened 55 anticancer metastasis drugs (50). It was demonstrated that the histology, molecular structure and function of the organoids remained highly consistent with those of the primary tumor tissues, thereby confirming the usefulness of their characteristics (50). When predicting patients' response to drugs with PDOs, the overall sensitivity was $100 \%$, the specificity was $93 \%$, the positive predictive value was $88 \%$ and the negative predictive value was $100 \%$ (50). Organoids cultivated from the patients' samples were thereby demonstrated to have predictive ability. In addition to the potential for personalized treatment, organoids can also fulfill a crucial role in drug development (50). Optimizing the simulation of how tumors are expected to react to treatment can be helpful in terms of accelerating the process of drug discovery, and even in terms of decreasing the dependence on animal experiments (50).

Gastrointestinal organoids provide a basic experimental model, superior to experimental animal models and conventional cell culture models in several aspects. As newly generated preclinical tumor models, the gastrointestinal cancer organoids have had a major role in the study of the biological characteristics and underlying molecular mechanism of gastrointestinal cancer and have been demonstrated to simulate the occurrence and development of tumors. There are promising prospects with their application in the development and screening of antitumor drugs, targeted tumor therapies and personalized medicine, and their combined application with PDXs (51).

\section{Summary and outlook}

Limitations. Recently, research on organoids has achieved considerable progress. However, limitations exist between theoretical in vitro studies and clinical applications due to the technical bottlenecks of the culture system. First, organoids mainly rely on animal-derived Matrigel or collagen I. Matrigel, or animal-derived Matrigel components, are complex and their quality is difficult to control (5). Exotic pathogens are also potential risk factors (52). In addition, the organoid system ignores the effects of stromal cells (52). The current existing organoid cultures are devoid of intestinal microbiota, vascular endothelial cells, neurons and immune cells, so their role in the physiology and the pathology of tumor processes does not come into consideration (52). Organoid is only supposed to reflect the physiological or pathological characteristics of local tissues, rather than the effects of systemic inflammatory responses and autonomic nerves (53). The recent emergence of a new organoid culture model has allowed the co-culturing of epithelial organoids and mesenchymal cells, which will enable the further study of the interactions between tumor and mesenchymal cells (54), and nerves and vascular tissues. Fong et al (55) co-cultured tumor cells derived from the PDX model of prostate cancer with osteoblasts, and the 3D model formed in this culture system was able to successfully maintain the proliferative activity of cells, and maintain the state of osteogenesis. Thus, this type of organoid model can be applied to observe the interaction between tumor cells and the tumor microenvironment, including stromal cells. However, additional research is required for further investigation of the organoid culture system. For example, the addition of stromal cells, adipocytes or lymphocytes would make the organoid culture system even more similar to the living tumor microenvironment, thereby providing a more ideal model for studying the association between the microenvironment and the tumor. Furthermore, organoids lack homogeneity (56). As the organoids are cultivated in 3D Matrigel, a certain differential effect is exerted by the Matrigel on their growth (5). Organoids on the periphery of Matrigel generally tend to be larger, whereas their size in the middle of Matrigel is smaller (56). This size heterogeneity creates a problem in terms of the quantification and analysis of high-flux organoid cells (56). Organoid transplants still face many challenges (57). Although these organoids composed of many cells can simulate the internal structure of real organs in certain aspects, other structural 
characteristics closely associated with the function and development of real organs cannot be simulated as of now, including the lack of a vascular system (57). Thus far, organoids would be better described as miniature and simple organ models, rather than being a 'reduced version' of the real organ (57). For example, a neurologist who used brain organoids to study the Zika virus considers that researchers have so far been unable to give organoids for immune systems the integrity they require to replicate exactly what happens in the body when screening drugs (58). Thus, organoid transplantations still face many challenges. In general, organoids cannot be successfully transplanted into mice in place of real organs due to their lack of vascular system and functionality $(56,57)$.

Perspective. Organoids have unique advantages in tumor research: i) They maintain high heterogeneity of tumor cells; ii) they maintain contact polarity between tumor cells and the microenvironment matrix to better simulate the tumor microenvironment in vivo; iii) organoid cultures derived from clinical tissue are highly efficient and time-saving and iv) tumor organoids also have the advantages of genetic manipulation of tumor cell lines and 3D complex-system characteristics of mouse PDX models (59).

However, further studies are required to optimize the culture techniques, as organoids derived from different human tissues may have the potential to promote relevant medical technologies; for example, establishing models of rare diseases in vitro, biological 3D printing, screening of patient's individual drugs and therapies, primary screening of toxicity and side effects, high-throughput drug screening, transplantation of organoids in vivo and the study of tissue regeneration. The future application of organoids will be broad and diverse, and it is hoped that the development of organoids will be an indispensable foundation for precision medicine in the future.

\section{Acknowledgements}

Not applicable.

\section{Funding}

The present review was supported by grants from the Natural Science Foundation of Jiangsu Province (grant no. BK20191223), the Key Program of Jiangsu Commission of Health (grant no. K2019024), the Young Medical Talents of Jiangsu (grant no. QNRC2016833), the Six Talent Peals Project of Jiangsu Province (grant no. WSW-039), the Six for One Project of Jiangsu Province (grant no. LGY2018093) and the Postgraduate Research \& Practice Innovation Program of Jiangsu Province (grant no. SJCX19_1175).

\section{Availability of data and materials}

Not applicable.

\section{Authors' contributions}

$\mathrm{XY}$ and $\mathrm{XX}$ wrote the draft of the manuscript. MW and $\mathrm{HZ}$ contributed to the conception of the work and organized the structure of the manuscript. DW revised the manuscript for important intellectual content. HZ and DW confirm the authenticity of all the raw data. All authors have read and approved the final manuscript.

\section{Ethics approval and consent to participate}

Not applicable.

\section{Patient consent for publication}

Not applicable.

\section{Competing interests}

The authors declare that they have no competing interests.

\section{References}

1. Lau H, Kranenburg O, Xiao $\mathrm{H}$ and Yu J: Organoid models of gastrointestinal cancers in basic and translational research. Nat Rev Gastroenterol Hepatol 17: 203-222, 2020.

2. Wei W, Zeng H, Zheng R, Zhang S, An L, Chen R, Wang S, Sun K, Matsuda T, Bray F and He J: Cancer registration in China and its role in cancer prevention and control. Lancet Oncol 21: e342-e349, 2020.

3. Gähwiler BH, Capogna M, Debanne D, McKinney RA and Thompson SM: Organotypic slice cultures: A technique has come of age. Trends Neurosci 20: 471 477, 1997.

4. Fatehullah A, Tan SH and Barker N: Organoids as an in vitro model of human development and disease. Nat Cell Biol 18: 246 254, 2016

5. Clevers H: Modeling development and disease with organoids. Cell 165: 1586-1597, 2016.

6. Dutta D, Heo I and Clevers H: Disease modeling in stem cell-derived 3D organoid systems. Trends Mol Med 23: 393-410, 2017.

7. Chen J,Lau BT, Andor N, Grimes SM,Handy C, Wood-Bouwens C and Ji HP: Single-cell transcriptome analysis identifies distinct cell types and niche signaling in a primary gastric organoid model. Sci Rep 9: 4536, 2019.

8. Hartl L, Huelsz-Prince G, van Zon J and Tans SJ: Apical constriction is necessary for crypt formation in small intestinal organoids. Dev Biol 450: 76-81, 2019.

9. Artegiani B, van Voorthuijsen L, Lindeboom RGH, Seinstra D, Heo I, Tapia P, López-Iglesias C, Postrach D, Dayton T, Oka R, et al: Probing the tumor suppressor function of BAP1 in CRISPR-engineered human liver organoids. Cell Stem Cell 24: 927-943.e6, 2019.

10. Matsuura T, Maru Y, Izumiya M, Hoshi D, Kato S, Ochiai M, Hori M, Yamamoto S, Tatsuno K, Imai T, et al: Organoid-based ex vivo reconstitution of Kras-driven pancreatic ductal carcinogenesis. Carcinogenesis 41: 490-501, 2020.

11. Shu $\mathrm{Y}$ and Chua CW: An organoid assay for long-term maintenance and propagation of mouse prostate luminal epithelial progenitors and cancer cells. Methods Mol Biol 1940: 231-254, 2019.

12. Gao D and Chen Y: Organoid development in cancer genome discovery. Curr Opin Genet Dev 30: 42-48, 2015.

13. Schumacher D, Andrieux G, Boehnke K, Keil M, Silvestri A, Silvestrov M, Keilholz U, Haybaeck J, Erdmann G, Sachse C, et al: Heterogeneous pathway activation and drug response modelled in colorectal-tumor-derived 3D cultures. PLoS Genet 15: e1008076, 2019.

14. Corrò C, Novellasdemunt L and Li VSW: A brief history of organoids. Am J Physiol Cell Physiol 319: C151-C165, 2020.

15. Sato T, Vries RG, Snippert HJ, van de Wetering M, Barker N, Stange DE, van Es JH, Abo A, Kujala P, Peters PJ and Clevers H: Single Lgr5 stem cells build crypt-villus structures in vitro without a mesenchymal niche. Nature 459: 262-265, 2009.

16. Neal J, Li X, Zhu J, Giangarra V, Grzeskowiak CL, Ju J, Liu IH, Chiou SH, Salahudeen AA, Smith AR, et al: Organoid modeling of the tumor immune microenvironment. Cell 175: 1972-1988.e16, 2018.

17. Wild CP, Weiderpass E and Stewart BW (eds.): World Cancer Report: Cancer Research for Cancer Prevention. World Health Organisation, Geneva, Switzerland, pp 23-33, 2020 
18. Liu HD, Xia BR, Jin MZ and Lou G: Organoid of ovarian cancer: genomic analysis and drug screening. Clinical and Translational Oncology 22: 1240-1251, 2020.

19. Bartfeld S, Bayram T, van de Wetering M, Huch M, Begthel H, Kujala P, Vries R, Peters PJ and Clevers H: In vitro expansion of human gastric epithelial stem cells and their responses to bacterial infection. Gastroenterology 148: 126-136.e6, 2015.

20. Bertaux-Skeirik N, Centeno J, Gao J, Gabre J and Zavros Y: Oncogenic transformation of human-derived gastric organoids. Methods Mol Biol 1576: 205-213, 2019.

21. Nagle PW, Plukker JTM, Muijs CT, van Luijk P and Coppes RP: Patient-derived tumor organoids for prediction of cancer treatment response. Semin Cancer Biol 53: 258-264, 2018.

22. Wenzel C, Riefke B, Gründemann S, Krebs A, Christian S, Prinz F Osterland M, Golfier S, Räse S, Ansari N, et al: 3D high-content screening for the identification of compounds that target cells in dormant tumor spheroid regions. Exp Cell Res 323: 131-143, 2014

23. Arena S, Corti G, Durinikova E, Montone M, Reilly NM, Russo M, Lorenzato A, Arcella P, Lazzari L, Rospo G, et al: A subset of colorectal cancers with cross-sensitivity to olaparib and oxaliplatin. Clin Cancer Res 26: 1372-1384, 2020.

24. Yao Y, Xu X, Yang L, Zhu J, Wan J, Shen L, Xia F, Fu G, Deng Y, Pan $\mathrm{M}$, et al: Patient-derived organoids predict chemoradiation responses of locally advanced rectal cancer. Cell Stem Cell 26: 17-26.e6, 2020.

25. Skardal A, Shupe T and Atala A: Organoid-on-a-chip and body-on-a-chip systems for drug screening and disease modeling. Drug Discov Today 21: 1399-1411, 2016.

26. Saito Y: Establishment of an organoid bank of biliary tract and pancreatic cancers and its application for personalized therapy and future treatment. J Gastroenterol Hepatol 34: 1906-1910, 2019.

27. van de Wetering M, Francies HE, Francis JM, Bounova G, Iorio F, Pronk A, van Houdt W, van Gorp J, Taylor-Weiner A, Kester L, et al: Prospective derivation of a living organoid biobank of colorectal cancer patients. Cell 161: 933-945, 2015.

28. Kondo J and Inoue MJC: Application of cancer organoid model for drug screening and personalized therapy. Cells 8: 470, 2019.

29. Broutier L, Mastrogiovanni G, Verstegen MM, Francies HE, Gavarró LM, Bradshaw CR, Allen GE, Arnes-Benito R, Sidorova O, Gaspersz MP, et al: Human primary liver cancer-derived organoid cultures for disease modeling and drug screening. Nat Med 23: 1424-1435, 2017.

30. Fong ELS, Toh TB, Lin QXX, Liu Z, Hooi L, Mohd Abdul Rashid MB, Benoukraf T, Chow EK, Huynh TH and Yu H: Generation of matched patient-derived xenograft in vitro-in vivo models using 3D macroporous hydrogels for the study of liver cancer. Biomaterials 159: 229-240, 2018.

31. Siegel RL, Miller KD and Jemal A: Cancer statistics, 2016. CA Cancer J Clin 66: 7-30, 2016.

32. Zhang HC and Kuo CJ: Personalizing pancreatic cancer organoids with hPSCs. Nat Med 21: 1249-1251, 2015.

33. Boj SF, Hwang CI, Baker LA, Chio II, Engle DD, Corbo V, Jager M, Ponz-Sarvise M, Tiriac H, Spector MS, et al: Organoid models of human and mouse ductal pancreatic cancer. Cell 160: 324-338, 2015

34. Patman G: Pancreatic cancer: From normal to metastases-a whole gamut of pancreatic organoids. Nat Rev Gastroenterol Hepatol 12: 61, 2015.

35. Huang L, Holtzinger A, Jagan I, BeGora M, Lohse I, Ngai N, Nostro C, Wang R, Muthuswamy LB, Crawford HC, et al: Ductal pancreatic cancer modeling and drug screening using human pluripotent stem cell- and patient-derived tumor organoids. Nat Med 21: 1364-1371, 2015.

36. Chio IIC, Jafarnejad SM, Ponz-Sarvise M, Park Y, Rivera K, Palm W, Wilson J, Sangar V, Hao Y, Öhlund D, et al: NRF2 promotes tumor maintenance by modulating mRNA translation in pancreatic cancer. Cell 166: 963-976, 2016.

37. Öhlund D, Handly-Santana A, Biffi G, Elyada E, Almeida AS, Ponz-Sarvise M, Corbo V, Oni TE, Hearn SA,Lee EJ, et al: Distinct populations of inflammatory fibroblasts and myofibroblasts in pancreatic cancer. J Exp Med 214: 579-596, 2017.

38. Lesina M, Kurkowski MU, Ludes K, Rose-John S, Treiber M, Klöppel G, Yoshimura A, Reindl W, Sipos B, Akira S, et al: Stat3/Socs 3 activation by IL-6 transsignaling promotes progression of pancreatic intraepithelial neoplasia and development of pancreatic cancer. Cancer Cell 19: 456-469, 2011.

39. Roe JS, Hwang CI, Somerville TDD, Milazzo JP, Lee EJ, Da Silva B, Maiorino L, Tiriac H, Young CM, Miyabayashi $\mathrm{K}$, et al: Enhancer reprogramming promotes pancreatic cancer metastasis. Cell 170: 875-888.e20, 2017.
40. Tiriac H, Bucobo J, Tzimas D, Grewel S, Lacomb JF, Rowehl LM, Nagula S, Wu M, Kim J, Sasson A, et al: Successful creation of pancreatic cancer organoids by means of EUS-guided fine-needle biopsy sampling for personalized cancer treatment. Gastrointest Endosc 87: 1474-1480, 2018.

41. Seino T, Kawasaki S, Shimokawa M, Tamagawa H, Toshimitsu K, Fujii M, Ohta Y, Matano M, Nanki K, Kawasaki K, et al: Human pancreatic tumor organoids reveal loss of stem cell niche factor dependence during disease progression. Cell Stem Cell 22: 454-467 2018.

42. Frappart PO, Walter K, Gout J, Beutel AK, Morawe M, Arnold F, Breunig M, Barth TF, Marienfeld R, Schulte L, et al: Pancreatic cancer-derived organoids-a disease modeling tool to predict drug response. United European Gastroenterol J 8: 594-606, 2020.

43. Múnera JO, Sundaram N, Rankin SA, Hill D, Watson C, Mahe M, Vallance JE, Shroyer NF, Sinagoga KL, Zarzoso-Lacoste A, et al: Differentiation of human pluripotent stem cells into colonic organoids via transient activation of BMP signaling. Cell Stem Cell 21:51-64,2017.

44. Webber JP, Spary LK, Sanders AJ, Chowdhury R, Jiang WG, Steadman R, Wymant J, Jones AT, Kynaston H, Mason MD, et al: Differentiation of tumour-promoting stromal myofibroblasts by cancer exosomes. Oncogene 34: 290-302, 2015.

45. Usui T, Sakurai M, Enjoji S, Kawasaki H, Umata K, Ohama T, Fujiwara N, Yabe R, Tsuji S, Yamawaki H, et al: Establishment of a novel model for anticancer drug resistance in three-dimensional primary culture of tumor microenvironment. Stem Cells Int 2016: 7053872, 2016.

46. Lannagan TRM, Lee YK, Wang T, Roper J, Bettington ML, Fennell L, Vrbanac L, Jonavicius L, Somashekar R, Gieniec K, et al: Genetic editing of colonic organoids provides a molecularly distinct and orthotopic preclinical model of serrated carcinogenesis. Gut 68: 684-692, 2019.

47. Nadauld LD, Garcia S, Natsoulis G, Bell JM, Miotke L, Hopmans ES, Xu H, Pai RK, Palm C, Regan JF, et al: Metastatic tumor evolution and organoid modeling implicate TGFBR 2 as a cancer driver in diffuse gastric cancer. Genome Biol 15: 428, 2014

48. Li X, Nadauld L, Ootani A, Corney DC, Pai RK, Gevaert O, Cantrell MA, Rack PG, Neal JT, Chan CW, et al: Oncogenic transformation of diverse gastrointestinal tissues in primary organoid culture. Nat Med 20: 769-777, 2014

49. Seidlitz T, Merker SR, Rothe A, Zakrzewski F, von Neubeck C, Grützmann K, Sommer U, Schweitzer C, Schölch S, Uhlemann $\mathrm{H}$, et al: Human gastric cancer modelling using organoids. Gut 68: 207-217, 2019.

50. Vlachogiannis G, Hedayat S, Vatsiou A, Jamin Y, Fernández-Mateos J, Khan K, Lampis A, Eason K, Huntingford I, Burke R, et al: Patient-derived organoids model treatment response of metastatic gastrointestinal cancers. Science 359: 920-926, 2018.

51. Singh A, Poling HM, Spence JR, Wells JM and Helmrath MA: Gastrointestinal organoids: A next-generation tool for modeling human development. Am J Physiol Gastrointest Liver Physiol 319: G375-G381, 2020

52. Xu H,Lyu X, Yi M,Zhao W, Song Y and Wu K: Organoid technology and applications in cancer research. J Hematol Oncol 11: 116, 2018

53. Foulke-Abel J, In J, Kovbasnjuk O, Zachos NC, Ettayebi K, Blutt SE, Hyser JM, Zeng XL, Crawford SE, Broughman JR, et al: Human enteroids as an ex-vivo model of host-pathogen interactions in the gastrointestinal tract. Exp Biol Med (Maywood) 239: 1124-1134, 2014

54. Sachs N, Tsukamoto Y, Kujala P, Peters PJ and Clevers H: Intestinal epithelial organoids fuse to form self-organizing tubes in floating collagen gels. Development 144: 1107-1112, 2017.

55. Fong EL, Wan X, Yang J, Morgado M, Mikos AG, Harrington DA, Navone NM and Farach-Carson MC: A 3D in vitro model of patient-derived prostate cancer xenograft for controlled interrogation of in vivo tumor-stromal interactions. Biomaterials 77: 164-172, 2016

56. Zhang S, Wan Z and Kamm RD: Vascularized organoids on a chip: Strategies for engineering organoids with functional vasculature. Lab Chip 21: 473-488, 2021.

57. Zhang C, Jin M, Zhao J, Chen J and Jin W: Organoid models of glioblastoma: Advances, applications and challenges. Am J Cancer Res 10: 2242-2257, 2020.

58. Date S and Sato T: Mini-gut organoids: Reconstitution of the stem cell niche. Annu Rev Cell Dev Biol 31: 269-289, 2015.

59. Kim J, Koo BK and Knoblich JA: Human organoids: Model systems for human biology and medicine. Nat Rev Mol Cell Biol 21: 571-584, 2020.

This work is licensed under a Creative Commons Attribution-NonCommercial-NoDerivatives 4.0 International (CC BY-NC-ND 4.0) License. 